\title{
Metastable Dark States Enable Ground State Depletion Microscopy of Nitrogen Vacancy Centers in Diamond with Diffraction- Unlimited Resolution
}

\author{
Kyu Young Han, ${ }^{\dagger,}$ Seong Keun Kim, ${ }^{\mp, \S}$ Christian Eggeling, ${ }^{*}{ }^{\dagger}$ and Stefan W. Hell ${ }^{*}{ }^{\dagger}$ \\ ${ }^{\dagger}$ Department of NanoBiophotonics, Max Planck Institute for Biophysical Chemistry, Am Fassberg 11, 37077 \\ Göttingen, Germany and ${ }^{*}$ Department of Chemistry and ${ }^{\S}$ Department of Biophysics and Chemical Biology, \\ Seoul National University, Seoul 151-747, Korea
}

\begin{abstract}
Current far-field optical nanoscopy schemes overcome the diffraction barrier by ensuring that adjacent features assume different states upon detection. Ideally, the transition between these states can be repeated endlessly and, if performed optically, with low levels of light. Here we report such optical switching, realized by pairing the luminescent triplet and a long-lived dark state of diamond color centers, enabling their imaging with a resolution $>10$ times beyond the diffraction barrier $(<20 \mathrm{~nm})$.
\end{abstract}

KEYWORDS Microscopy, color center, diamond, subdiffraction imaging, ground state depletion, dark state

2 hroughout the 20th century it was widely accepted that the resolution of lens-based light microscopy is fundamentally limited by diffraction. ${ }^{1}$ However, in the mid 1990s it was shown that in fluorescence imaging the diffraction limit can be overcome by using a molecular mechanism that keeps the fluorophore dark even when it is exposed to excitation light. ${ }^{2,3}$ Keeping fluorophores dark facilitates recording nearby fluorescent features in a sequential manner, ${ }^{2-8}$ thus making them distinguishable. For example, in stimulated emission (STED) microscopy, ${ }^{2,9}$ the fluorophore is transiently confined to its dark ground state by disallowing the occupation of its fluorescent state. In ground state depletion (GSD) microscopy ${ }^{3,10}$ and in the single molecule switching localization methods called GSDIM (standing for GSD followed by individual molecule return), ${ }^{1-13}$ the fluorophore is transiently confined to a dark (triplet or redox) state. In the variant called SSIM (saturable structured illumination microscopy), ${ }^{14,15}$ the ground state is depleted by confining the fluorophore to an excited state in which the excitation light may not elicit any further photon. Cis-trans photoisomerization ${ }^{16,17}$ or similar photoreactions $^{5-7,18}$ can also be employed as on-off transitions. As a matter of fact, the utilization of on-off transitions has been the enabling element in the conversion of farfield optical microscopy into methods resolving at the nanoscale. ${ }^{8,19}$

*To whom correspondence should be addressed, ceggeli@gwdg.de and shell@ gwdg.de.

Received for review: 06/18/2010

Published on Web: 07/22/2010
Therefore, current nanoscopy methods are more or less affected by the limited total number of on-off cycles the fluorophore can accommodate before bleaching. ${ }^{8,19}$ The total number of cycles is particularly critical for the RESOLFT (reversible saturable optical fluorescence transition) methods, encompassing STED, GSD, and SSIM, in which the on-off transition is predefined in space by a spatially structured light intensity distribution featuring one or more local minima or zeros

A notable exception is presented by negatively charged nitrogen-vacancy $\left(\mathrm{NV}^{-}\right)$color centers in diamond. ${ }^{20-23} \mathrm{Con}-$ sisting of substituted nitrogen with a neighboring vacancy (Figure 1a) this paramagnetic emitter offers facile accessibility to spin manipulation ${ }^{20,21}$ and remarkable photostability. ${ }^{20-23}$ Its maximum photon absorption is centered at $560 \mathrm{~nm}$, leading from the triplet ground to the triplet luminescent state with the zero-phonon line at $637 \mathrm{~nm}$. The resulting luminescence is Stokes-shifted to 600-850 nm and has a lifetime of $\sim 12 \mathrm{~ns}^{21,22}$ The remarkable photostability of $\mathrm{NV}^{-}$color centers has enabled STED microscopy ${ }^{23,24}$ with single digit nanometer resolution, as well as a variant of GSD whereby the ground state was depleted by a high intensity beam of $532 \mathrm{~nm}$ wavelength pumping the centers to the luminescent state. ${ }^{25}$ However, by operating mainly with the basic triplet states of the centers, these approaches required optical intensities up to several $\mathrm{GW} / \mathrm{cm}^{2}$ to attain a resolution $<10 \mathrm{~nm}$. Here, we report a fatigue-free luminescence switch that is all-optically controlled in both directions and operates at several orders of magnitude lower power. Moreover, we show that by utilizing a singlet-triplet transition, i.e., a "spin flip”, this switching mechanism enables classical low-power 


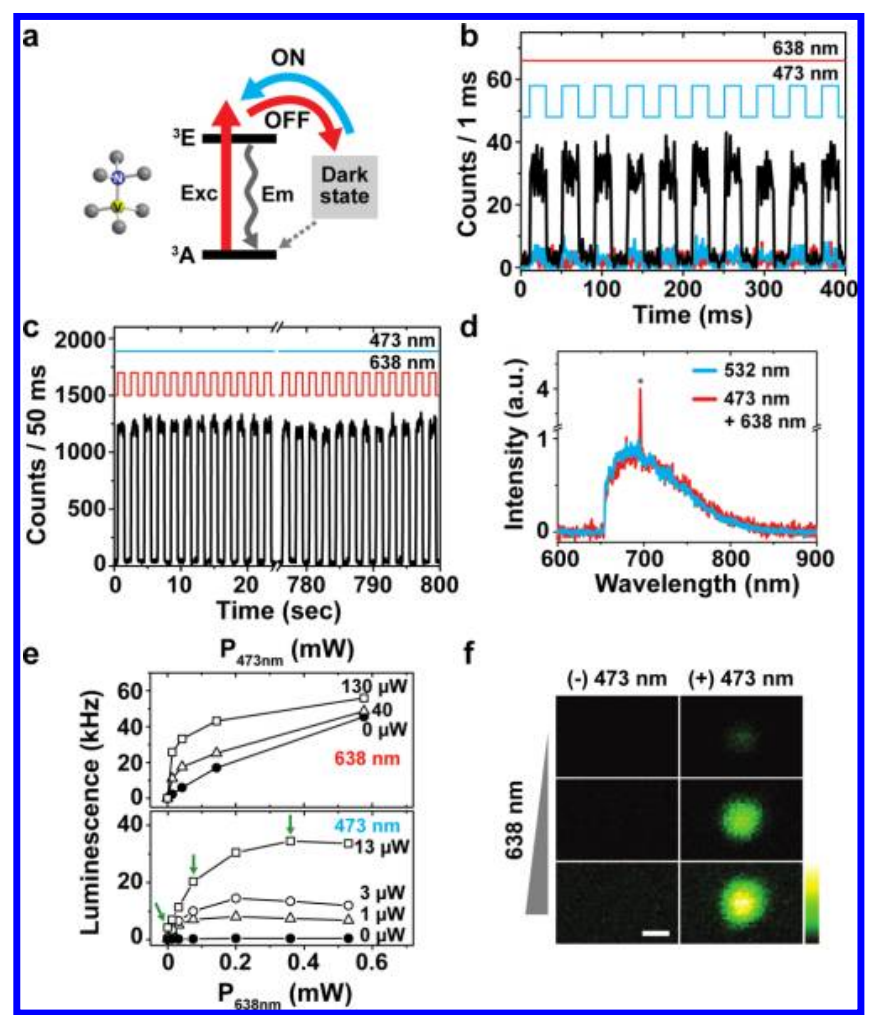

FIGURE 1. Low-power on-off switching of a single $\mathrm{NV}^{-}$center luminescence in diamond. (a) Energy diagram and structure in diamond network (inset) of negatively charged NV center with lightinduced (solid lines), radiative (wavy lines), and nonradiative transitions (dotted lines). (b, c) Continuous on-off cycling of the $\mathrm{NV}^{-}$ luminescence with $473 \mathrm{~nm}(12 \mu \mathrm{W})$ and $638 \mathrm{~nm}(200 \mu \mathrm{W})$ laser light: luminescence follows the modulation of $473 \mathrm{~nm}$ (b) or $638 \mathrm{~nm}$ light (c) and negligible luminescence is detected with only one laser switched on (blue and red lines in (b)). (d) Emission spectra of a single $\mathrm{NV}^{-}$center at room temperature following sole $532 \mathrm{~nm}$ and combined 473 and $638 \mathrm{~nm}$ illumination (asterisks, Raman scattering peak from diamond). (e) Dependence of the luminescence from a single $\mathrm{NV}^{-}$on the laser powers of the combined 473 and $638 \mathrm{~nm}$ illumination. (f) Single-emitter all-optical AND gate: Confocal luminescence image of a single $\mathrm{NV}^{-}$center with (right) and without (left) $13 \mu \mathrm{W}$ of $473 \mathrm{~nm}$ light and increasing power of $638 \mathrm{~nm}$ light (upper, $0 \mu \mathrm{W}$; middle, $75 \mu \mathrm{W}$; lower, $360 \mu \mathrm{W}$ as also marked by green arrows in (e)). Scale bar represents $200 \mathrm{~nm}$.

GSD microscopy, the demonstration of which at ultrahigh resolution (<20 $\mathrm{nm}$ ) has remained elusive since its proposal in $1994 .^{3,26}$

The key to establishing a low light level nanoscopy method is to realize the on-off switch with long-lived dark states, such as triplet, radical ion, or conformational states. ${ }^{3,16}$ Figure 1 demonstrates fatigue-free switching of the luminescence ability of $\mathrm{NV}^{-}$centers by low levels of light. Using a home-built confocal microscope, we continuously excited a single $\mathrm{NV}^{-}$center in type IIa diamond grown by chemical vapor deposition with $200 \mu \mathrm{W}$ of $638 \mathrm{~nm}$ red laser light, and periodically added $12 \mu \mathrm{W}$ of $473 \mathrm{~nm}$ blue laser light (Figure $1 \mathrm{~b})$ or vice versa (Figure 1c). Bright luminescence is detectable only when irradiating with both blue and red light, while neither the red nor the blue light irradiation alone elicits a strong signal. The on-off luminescence cycling is reproduc- ible and follows the modulation of the laser light, showing no signs of photobleaching or random blinking. The on-off switching is instantaneous within the temporal resolution of 100 ns provided by our experiment (Figure S1 in Supporting Information). The coirradiation by red and blue light generates an emission spectrum from a single $\mathrm{NV}^{-}$center (Figure $1 \mathrm{~d})$, which is indistinguishable from that generated by the commonly used excitation at $532 \mathrm{~nm}$.

The luminescence increases with increasing power of either blue or red light, saturating at $>200-300 \mu \mathrm{W}$ (Figure 1e). Switching luminescence was also possible with slightly different wavelengths, e.g., 491 and $647 \mathrm{~nm}$, but less pronounced when applying longer wavelengths such as 671 nm (Figure S2 in Supporting Information). The switching can be perceived as a single quantum emitter all-optical AND gate capable of processing information. Moreover, the power of the output light can be tuned by changing the optical power of the input (Figure $1 \mathrm{f}$ ). The switching is rather distinct from most other photoinduced on-off switches where the two input beams perform either on- or off-switching. ${ }^{27}$ For example, reported photochromism of NV luminescence implied cycling between the neutral $\mathrm{NV}^{0}$ and the charged $\mathrm{NV}^{-}$form of the very same center, with distinct emission bands at $\sim 600$ and $700 \mathrm{~nm}$, respectively. ${ }^{28}$ We did not detect $N V^{0}$ luminescence when applying the $473 \mathrm{~nm}$ light only or both the 473 and $638 \mathrm{~nm}$ illumination (Figure S3 in Supporting Information).

In addition to its bright triplet system having a ground $\left({ }^{3} \mathrm{~A}\right)$ and a luminescent state $\left({ }^{3} \mathrm{E}\right)$, the $\mathrm{NV}^{-}$center has multiple dark (singlet) states (Figure 1a). ${ }^{29}$ Previous experiments on $\mathrm{NV}^{-}$defect centers in diamond revealed that the dark state transitions may be induced optically. ${ }^{30-33}$ At cryogenic temperatures the luminescence of these centers in type Ib diamond following $638 \mathrm{~nm}$ excitation could be enhanced by $488 \mathrm{~nm}$ light. ${ }^{30}$ An explanation is that the dark state is emptied by blue light via transitions to higher states. ${ }^{30}$ Fluorescence correlation experiments on single centers revealed an intensity-dependent increase of the dark state transition rates following $532 \mathrm{~nm}$ excitation. ${ }^{31,32}$ On the basis of these results, we propose that, while the red light may excite the $\mathrm{NV}^{-}$center to ${ }^{3} \mathrm{E}$, it also efficiently transfers the $\mathrm{NV}^{-}$to a metastable dark state, thereby depleting its ground state $\left({ }^{3} \mathrm{~A}\right)$. Conversely, excitation to ${ }^{3} \mathrm{E}$ is comparatively inefficient for blue light $<490 \mathrm{~nm}$, but this wavelength effectively depopulates the dark state. Therefore, only the simultaneous irradiation by blue and red light yields strong luminescence.

This model is supported by several findings. Figure $2 \mathrm{a}$ depicts a pump-probe measurement on a single $\mathrm{NV}^{-}$center applying interlaced trains of 473 and $638 \mathrm{~nm}$ pulses. A 80 $\mu \mathrm{s}$ long $473 \mathrm{~nm}$ pulse $(20 \mu \mathrm{W})$ was followed by a $80 \mu \mathrm{s}$ long $638 \mathrm{~nm}$ pulse $(200 \mu \mathrm{W})$ with $40 \mu \mathrm{s}$ time delay; this sequence was repeated 50000 times every $256 \mu \mathrm{s}$. The signal in Figure 2 a results from integration over the whole sequence. Our model suggests that excitation by $638 \mathrm{~nm}$ light leads to 


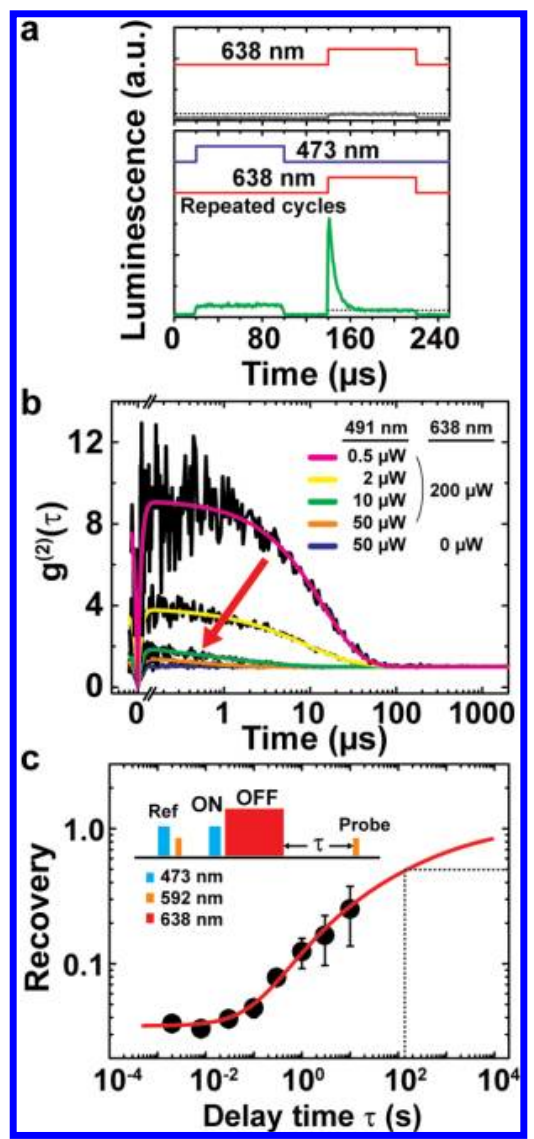

FIGURE 2. On-off mechanism. (a) Time-dependent luminescence of a single $\mathrm{NV}^{-}$center following a 50000 times repeated pulse sequence of 473 and $638 \mathrm{~nm}$ light: 473 and $638 \mathrm{~nm}$ pulses (lower panel) and just $638 \mathrm{~nm}$ (upper panel). Dotted lines represent the level of scattering background signal at $638 \mathrm{~nm}$ light. (b) Autocorrelation of luminescence time traces of a single $\mathrm{NV}^{-}$for different power levels of 491 and $638 \mathrm{~nm}$ light. Dark state population decreases with increasing power of $491 \mathrm{~nm}$ light indicated by a red arrow. (c) Lifetime of the dark state for a single $\mathrm{NV}^{-}$center determined by a repeated pump-probe cycle of 473, 638, and 592 $\mathrm{nm}$ light (inset). Exponential fitting (red line) yields a decay time of $150 \mathrm{~s}$.

cycling between ground state ${ }^{3} \mathrm{~A}$ and luminescent state ${ }^{3} \mathrm{E}$, but after some cycles the center ends up in a dark state. The luminescence decays rapidly with extended exposure to 638 $\mathrm{nm}$ light due to the increasing probability of the center to be transferred to a dark state. The $638 \mathrm{~nm}$ pulse produces a notable signal only if it follows a $473 \mathrm{~nm}$ counterpart. With no $473 \mathrm{~nm}$ light, luminescence occurs only during the first pulse of the $638 \mathrm{~nm}$ sequence, resulting in a negligible signal (upper panel in Figure 2a). The luminescence detected during the $473 \mathrm{~nm}$ pulse is only slightly above background, because excitation to ${ }^{3} \mathrm{E}$ is inefficient at this wavelength and the applied power and also because a single transition from the dark to the luminescent state (on-switching) of a center may produce just a single photon.

We analyzed the temporal structure of the luminescence of a single center by autocorrelation. Figure $2 \mathrm{~b}$ shows the results from luminescence time traces following $200 \mu \mathrm{W}$ of $638 \mathrm{~nm}$ and different power levels of $491 \mathrm{~nm}$ light. (The 491 $\mathrm{nm}$ laser was used because it was less noisy than the one at $473 \mathrm{~nm}$.) The autocorrelation curve shows a steep rise, i.e., an antibunching term with a characteristic time of $\sim 10-20$ ns stemming from the transitions between the ${ }^{3} \mathrm{~A}$ and the ${ }^{3} \mathrm{E}$, and a decay reflecting photon bunches in the $10 \mu \mathrm{s}$ range due to dark state excursions. ${ }^{31,32}$ The amplitude and the decay time of the bunching term correlate with the population and with the depopulation rate of the dark state, respectively. In agreement with our model, the dark state population decreases and the depopulation rate increases with increasing power of blue light.

Our model only holds for a fairly stable dark state with a rather long relaxation time. In this case, depopulation of the dark state mainly occurs optically, but not spontaneously. The luminescence detected at different time spans after turning off the center by red light indicates a slow (>150 s) recovery (Figure 2c). The recovery rate is by far weaker than the optically driven rates of about $1 \mu$ s derived from the autocorrelation data of Figure $2 \mathrm{~b}$ or from previous reports. ${ }^{30-33}$ In any case, in conjunction with the stable ground state ${ }^{3} \mathrm{~A}$, the metastable dark state forms a nearly bistable luminescence switch that can be operated at comparatively low optical power. Clearly, such an all-optical switch enables farfield optical imaging of these centers with diffractionunlimited spatial resolution. ${ }^{3,8-10,16}$

In an implementation of the GSD concept, all emitters covered by a focused excitation beam are transiently switched off, except at a sub-diffraction-sized region. To this end, the focal spot of the excitation beam is covered, for example, by a doughnut-shaped beam of red "switch-off" light transferring the center to a long-lived dark state. The obtained resolution can be quantified by the spatial range in the focal plane in which the centers are "on". ${ }^{4}$ Scaling as $\sim 1 /\left(1+I / I_{\mathrm{S}}\right)^{1 / 2}$, the full-width-at-half-maximum (FWHM) ${ }^{8,9,16}$ of this area can in principle be reduced to subnanometer dimensions. $I$ is the intensity of the red light at the doughnut maximum and $I_{S}$ is a characteristic intensity giving the value at which the probability of the $\mathrm{NV}^{-}$to emit is reduced by half. $I_{\mathrm{S}}$ scales inversely both with the lifetime of the states involved and with the action cross section of the optical switch-off transition. Considering the doughnut area, the expression can be rewritten as $1 /\left(1+P / P_{\mathrm{S}}\right)^{1 / 2}$, with $P$ and $P_{\mathrm{s}}$ denoting the applied and characteristic power, respectively, which are more easily measured.

We first quantified the luminescence of a single $\mathrm{NV}^{-}$ center following the application of a $647 \mathrm{~nm}$ turn-off pulse (Figure S4 in Supporting Information), using a regularly focused beam for GSD. Measuring the "on" state probability of a center, which is equivalent to probing the residual luminescence, was performed by a short pulse (10 $\mu \mathrm{s})$ of $592 \mathrm{~nm}$ read-out light $(20 \mu \mathrm{W})$. This wavelength elicits luminescence but alters the dark state population much less than the green or blue light (Figure S4 in Supporting Information). Additionally, it facilitates depletion of the luminescent state down to $1-3 \%$ (Figure S5 


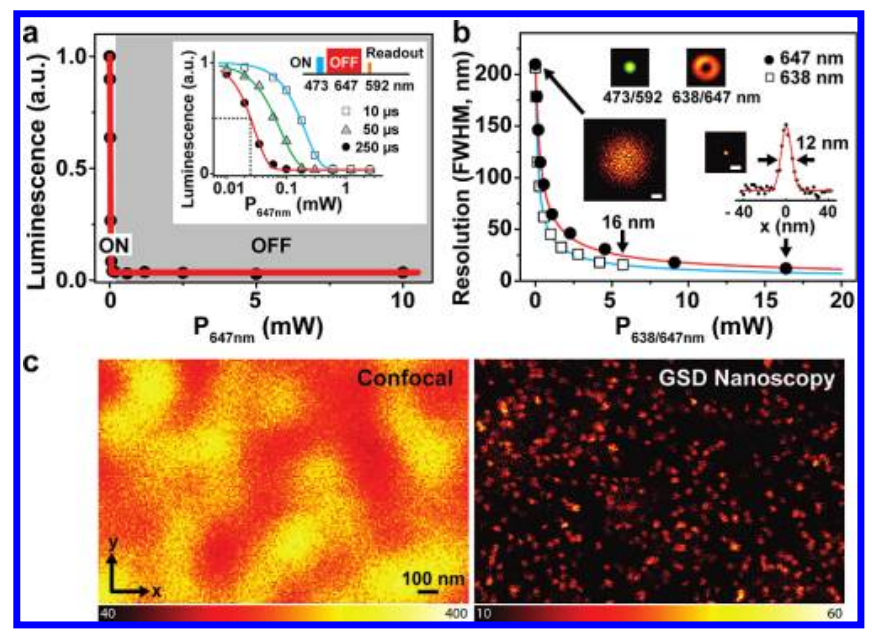

FIGURE 3. Ground state depletion (GSD) nanoscopy. (a) A nearly perfect luminescence switch: disallowance of the luminescent state (off-switch) of a single NV center with increasing power of $647 \mathrm{~nm}$ light. The residual luminescence was read out by $592 \mathrm{~nm}$ light following a pulse sequence of 473 and $647 \mathrm{~nm}$ light (inset). The power $P_{\mathrm{s}}$ needed to switch off half the NV luminescence (dotted line) depends on the length of the red pulse (inset). The data exhibit a nearly "rectangular" all-optical off-switching on a linear scale; details are inferred from a log scale (inset). (b) Scaling of resolution to subdiffraction dimensions (down to $12 \mathrm{~nm}$ ) by increasing power levels of the 638 or $647 \mathrm{~nm}$ light, probed by imaging a single NV center (inset). A doughnut-shaped intensity distribution of the red light was applied (inset). Scale bar represents $50 \mathrm{~nm}$. (c) Confocal (left) and GSD nanoscopy (right) images of NV color centers in bulk diamond in an area $1.8 \mu \mathrm{m} \times$ $1.2 \mu \mathrm{m}$ (dwell time, $6 \mathrm{~ms}$; pixel size, $5 \mathrm{~nm}$ ).

in Supporting Information). A $473 \mathrm{~nm}$ light pulse establishes the initial population of the ground state (Figure S6 in Supporting Information) before the next turn-off pulse takes action (inset Figure 3a). Figure 3 a shows that the luminescence steeply decreases with increasing power of the $647 \mathrm{~nm}$ light, demonstrating that the ability of the center to emit can be switched off by light.

$P_{\mathrm{s}}$ is in the range of $25-200 \mu \mathrm{W}$ depending on the length of the GSD pulse (inset Figure 3a) and on the area of the focal doughnut. The used power and the concomitant intensity are by about 1000-fold lower than for switching off the fluorescence of a typical organic dye by stimulated emission. ${ }^{9}$ If the duration of the applied pulses is at least about 3 times shorter than the lifetime of the dark state, the level of disallowance of the bright state depends only on the number of photons in the pulse. Therefore the pulse duration can be chosen such that the image acquisition time can be balanced against the GSD efficiency.

The same pump-probe cycle was used for obtaining GSD scanning nanoscopy images. We overlaid a doughnutshaped distribution of the red light for GSD (inset Figure 3b) to regularly focused spots of $473 \mathrm{~nm}$ switch-on and $592 \mathrm{~nm}$ read-out light. The subdiffraction resolution was established from the FWHM of single $\mathrm{NV}^{-}$center images for different power levels of the red light for GSD (Figure 3b). We applied a $500 \mu$ s long pulse of the red light and repeated the pulse sequence 50 times to integrate signal.

GSD allowed tuning the resolution from the diffraction limit $(210 \mathrm{~nm})$ down to $12 \mathrm{~nm}$. The latter was attained at $P=16 \mathrm{~mW}$ corresponding to a peak intensity of $I=12$ $\mathrm{MW} / \mathrm{cm}^{2}$ in our doughnut pulse. The maximum resolution was limited only by the finite power of the laser employed for GSD. The FWHM clearly follows the $1 /\left(1+P / P_{S}\right)^{1 / 2}$ law. Applying shorter GSD pulses may shorten the acquisition time, but due to the elevated $P_{\mathrm{s}}$ the shorter pulses also increase the power needed to achieve the same resolution (inset Figure 3a). Furthermore, due to the lower $P_{\mathrm{S}}$ (Figure S7 in Supporting Information), light at $638 \mathrm{~nm}$ wavelength was slightly more efficient than that at $647 \mathrm{~nm}$. Nevertheless, we used the $647 \mathrm{~nm}$ laser for further imaging because it supplied more power. Figure 3c compares images of $\mathrm{NV}^{-}$centers in diamond taken with standard confocal and GSD nanoscopy, where we used short GSD laser pulses ( $50 \mu \mathrm{s}, 26 \mathrm{~mW}, 647 \mathrm{~nm}$ ) to accelerate image acquisition. Clearly, GSD but not confocal microscopy resolves the $\mathrm{NV}^{-}$centers.

In conclusion, transiently inhibiting luminescence by GSD through transferring the $\mathrm{NV}^{-}$to a metastable dark state and measuring the luminescence (with a separate pulse) of those $\mathrm{NV}^{-}$that remain in the bright state is an attractive alternative to STED $^{23}$ and previous GSD variants. ${ }^{25}$ While we used three wavelengths (initialization, GSD and read-out laser), the GSD approach described herein does not need any data processing to obtain the image; it relies just on the judicious exploitation of a transition between a luminescence "on" (triplet) and an "off" (singlet) state. The raw data obtained is the final image. For the same reason, GSD nanoscopy of $\mathrm{NV}^{-}$ centers should be readily combined with far-field optical magnetic imaging on the nanoscale. ${ }^{34,35}$ Reduction to the use of only two instead of three laser wavelengths would be feasible, since the very same laser (for example, blue or green light) could perform both initialization and readout. However, in this case the read-out is accompanied by a much stronger reduction of the dark state population, limiting the read-out signal. In principle, we could have also adopted blue light for initialization and red light for GSD as well as read-out. The reason why we applied the third laser (592 nm) was that when using $638 / 647 \mathrm{~nm}$ for read out we observed Raman scattering from bulk diamond. Another issue that may influence the performance of GSD nanoscopy using $\mathrm{NV}^{-}$centers is the type of sample. For example, the dark state lifetime may be influenced by the local nitrogen concentration, which is much higher in, for example, type Ib diamonds, or by surface proximity effects such as in NV-nanodiamonds. Importantly, since it uses a much lower power than the other methods, our GSD approach is more easily parallelized with multiple intensity minima and a camera. ${ }^{14-16}$ The GSD-type optical switching reported herein should 
also allow the implementation of a variant of GSDIM ${ }^{11}$ or PALM/STORM, ${ }^{5-7}$ i.e., methods that switch the single emitters stochastically in space followed by localization with a pixilated detector. Thus, our findings not only demonstrate the potential of $\mathrm{NV}^{-}$for turning far-field optical nanoscopy methods into practice but also emphasize the transition between two quantum states, here a bright and a dark state, as the key element enabling this radical change in optical microscopy resolution.

Acknowledgment. We thank our colleagues A. Schönle for support with Imspector software and D. Wildanger, E. Rittweger, and J. Maze (Harvard University) for fruitful discussions. We also thank F. Jelezko (Stuttgart University) and D. Twitchen (Element 6) for providing the diamond samples. Part of this work was supported by the WCU program (R31-10032) of the NRF and by the Gottfried Wilhelm Leibniz Program of the Deutsche Forschungsgemeinschaft.

Supporting Information Available. Detailed experimental methods and figures showing luminescence switching. This material is available free of charge via the Internet at http://pubs.acs.org.

\section{REFERENCES AND NOTES}

(1) Abbe, E. Arch. Mikrosk. Anat. 1873, 9, 413-468.

(2) Hell, S. W.; Wichmann, J. Opt. Lett. 1994, 19 (11), 780-782.

(3) Hell, S. W.; Kroug, M. Appl. Phys. B: Laser Opt. 1995, 60, 495497.

(4) Hell, S. W.; Jakobs, S.; Kastrup, L. Appl. Phvs. A: Mater. Sci. Process. 2003, 77, 859-860.

(5) Betzig, E.; Patterson, G. H.; Sougrat, R.; Lindwasser, O. W.; Olenych, S.; Bonifacino, J. S.; Davidson, M. W.; LippincottSchwartz, J.; Hess, H. F. Science 2006, 313 (5793), 1642-1645.

(6) Rust, M. J.; Bates, M.; Zhuang, X. W. Nat. Methods 2006, 3, 793 795.

(7) Hess, S. T.; Girirajan, T. P. K.; Mason, M. D. Biophys. I. 2006, 91 (11), 4258-4272.

(8) Hell, S. W. Science 2007, 316 (5828), 1153-1158.

(9) Westphal, V.; Hell, S. W. Phvs. Rev. Lett. 2005, 94, 143903.

(10) Bretschneider, S.; Eggeling, C.; Hell, S. W. Phys. Rev. Lett. 2007, 98 (21), 218103.

(11) Folling, J.; Bossi, M.; Bock, H.; Medda, R.; Wurm, C. A.; Hein, B.; Jakobs, S.; Eggeling, C.; Hell, S. W. Nat. Methods 2008, 5, 943945.

(12) Heilemann, M.; van de Linde, S.; Schuttpelz, M.; Kasper, R.; Seefeldt, B.; Mukherjee, A.; Tinnefeld, P.; Sauer, M. Angew. Chem. Int. Ed. 2008, 47, 6172-6176.
(13) Vogelsang, J.; Cordes, T.; Forthmann, C.; Steinhauer, C.; Tinnefeld, P. Proc. Natl. Acad. Sci. U.S.A. 2009, 106 (20), 8107-8112.

(14) Heintzmann, R.; Jovin, T. M.; Cremer, C. L.Opt. Soc. Am. A 2002, 19 (8), 1599-1609.

(15) Gustafsson, M. G. L. Proc. Natl. Acad. Sci. U.S.A. 2005, 102 (37), $13081-13086$.

(16) Hell, S. W. Nat. Biotechnol. 2003, 21 (11), 1347-1355.

(17) Hofmann, M.; Eggeling, C.; Jakobs, S.; Hell, S. W. Proc. Natl. Acad. Sci. U.S.A. 2005, 102 (49), 17565-17569.

(18) Bossi, M.; Foelling, J.; Dyba, M.; Westphal, V.; Hell, S. W. New I. Phvs. 2006, 8, 275.

(19) Hell, S. W. Nat. Methods 2009, 6 (1), 24-32.

(20) Gruber, A.; Drabenstedt, A.; Tietz, C.; Fleury, L.; Wrachtrup, J.; vonBorczyskowski, C. Science 1997, 276, 2012-2014.

(21) Jelezko, F.; Wrachtrup, J. Phvs. Status Solidi A 2006, 203, 3207 3225 .

(22) Fu, C. C.; Lee, H. Y.; Chen, K.; Lim, T. S.; Wu, H. Y.; Lin, P. K.; Wei, P. K.; Tsao, P. H.; Chang, H. C.; Fann, W. Proc. Natl. Acad. Sci. U.S.A. 2007, 104 (3), 727-732.

(23) Rittweger, E.; Han, K. Y.; Irvine, S. E.; Eggeling, C.; Hell, S. W. Nat. Photonics 2009, 3, 144-147.

(24) Han, K. Y.; Willig, K. I.; Rittweger, E.; Jelezko, F.; Eggeling, C.; Hell, S. W. Nano Lett. 2009, 9 (9), 3323-3329.

(25) Rittweger, E.; Wildanger, D.; Hell, S. W. Europhys. Lett. 2009, 86, 14001-p114001.p6

(26) Dose, V. Europhvs. Lett. 2009, 86, 10000.

(27) Irie, M.; Fukaminato, T.; Sasaki, T.; Tamai, N.; Kawai, T. Nature 2002, 420 (6917), 759-760.

(28) Gaebel, T.; Domhan, M.; Wittmann, C.; Popa, I.; Jelezko, F.; Rabeau, J.; Greentree, A.; Prawer, S.; Trajkov, E.; Hemmer, P. R.; Wrachtrup, J. Appl. Phvs. B: Laser Opt. 2006, 82 (2), 243-246.

(29) Larsson, J. A.; Delaney, P. Phvs. Rev. B 2008, 77 (16), 165201.

(30) Drabenstedt, A.; Fleury, L.; Tietz, C.; Jelezko, F.; Kilin, S.; Nizovtzev, A.; Wrachtrup, J. Phys. Rev. B: Condens. Matter 1999, 60 (16), 11503-11508.

(31) Kurtsiefer, C.; Mayer, S.; Zarda, P.; Weinfurter, H. Phvs. Rev. Lett. 2000, 85 (2), 290-293.

(32) Beveratos, A.; Brouri, R.; Poizat, J. P.; Grangier, P., Bunching and antibunching from single NV color centers in diamond. In Quantum Communication, Computing, and Measurement; Tombesi, P., Hirota, O., Eds.; Kluwer: New York, 2001; Vol. 3, pp 261-267.

(33) Jelezko, F.; Popa, I.; Gruber, A.; Tietz, C.; Wrachtrup, J.; Nizovtsev, A.; Kilin, S. Appl. Phys. Lett. 2002, 81 (12), 2160-2162.

(34) Balasubramanian, G.; Chan, I. Y.; Kolesov, R.; Al-Hmoud, M.; Tisler, J.; Shin, C.; Kim, C.; Wojcik, A.; Hemmer, P. R.; Krueger, A.; Hanke, T.; Leitenstorfer, A.; Bratschitsch, R.; Jelezko, F.; Wrachtrup, J. Nature 2008, 455, 648-U46.

(35) Maze, J. R.; Stanwix, P. L.; Hodges, J. S.; Hong, S.; Taylor, J. M.; Cappellaro, P.; Jiang, L.; Dutt, M. V. G.; Togan, E.; Zibrov, A. S.; Yacoby, A.; Walsworth, R. L.; Lukin, M. D. Nature 2008, 455, 644U41 